\title{
A LIAPUNOV FUNCTION FOR THREE-DIMENSIONAL FEEDBACK SYSTEMS
}

\author{
JIANG JI-FA
}

(Communicated by Kenneth R. Meyer)

\begin{abstract}
For a three-dimensional model of a positive feedback loop, Selgrade $[11,12]$ proved that every positive-time trajectory in the nonnegative orthant converges. Hirsch [6] gave another proof of this result under slightly different assumptions. This paper provides a new proof of Selgrade's result that is much shorter and presents a generalization that can be applied to positive and negative feedback loops and other systems.
\end{abstract}

\section{INTRODUCTION}

Consider the system of differential equations

$$
\dot{x}=-a x+f(z) \quad \dot{y}=x-b y \quad \dot{z}=y-c z,
$$

where $a, b$, and $c$ are positive constants, and $x, y$, and $z$ are nonnegative variables. Many authors have studied this model (see [1-6, 10-13]). If $f$ is $C^{1}$ and $f^{\prime} \geq 0$, then system (1) describes a positive feedback loop; if $f$ is $C^{1}$ and $f^{\prime} \leq 0$, then system (1) describes a negative feedback loop. Selgrade $[11,12]$ studied the positive feedback system (1) and proved the following main theorem:

If (1) satisfies the following conditions for $z \geq 0$ :

(A1) $f(z)>0$ if $z \neq 0$;

(A2) $f$ is $C^{1}$ and $f^{\prime}(z) \geq 0$;

(A3) $f(z)$ is bounded, and

(A4) the equilibria are finite in number;

then every positive-time trajectory converges to an equilibrium (see [11, Theorems 9.1, 10.2]). Using the order-preserving properties of cooperative systems and the first Čech cohomology group, Hirsch [6] provided another proof of Selgrade's theorem under slightly different assumptions (see [6, pp. 104-105]).

The purpose of this paper is to propose a new simpler proof and a generalization of Selgrade's theorem. Actually, we shall present a criterion for convergence of solutions of (1). This criterion can be applied to positive feedback loop (see

Received by the editors August 24, 1990.

1991 Mathematics Subject Classification. Primary 34C11; Secondary 92A09.

Key words and phrases. Positive and negative feedback systems, convergence, Liapunov function.

This research was supported in part by the National Natural Science Foundation of China. 
Corollary 2), negative feedback loop (see Corollaries 3, 4) and other systems (see Remark 4). The method employed here is based on an idea of Liapunov to construct a function that decreases along solutions with increasing time (see [7-9]).

\section{THE MAIN RESULTS}

Let $S_{1}=a+b+c, S_{2}=a b+b c+c a$, and $S_{3}=a b c$ where $a, b$, and $c$ are positive. Denote by $\mathbf{R}_{+}^{3}$ the nonnegative orthant of $\mathbf{R}^{3}$.

In this paper, we shall prove the following results.

Theorem 1. Assume that $f$ is $C^{1}$ on $[0, \infty)$ and that $\mathbf{R}_{+}^{3}$ is positively invariant for (1). If $S_{1} S_{2}-S_{3}+f^{\prime}(z)>0$ for $z \geq 0$, then every bounded solution of (1) converges to an equilibrium.

Corollary 2. Suppose that (1) satisfies assumptions (A1) and (A2). Then every solution of (1) converges to an equilibrium if and only if $f\left(z_{k}\right) \leq S_{3} z_{k}$ for some sequences $z_{k} \rightarrow \infty$.

If $f(z)=\left(1+z^{m}\right)^{-1}$ for $m \geq 1$, the system (1) is a model of a negative feedback cellular control process studied in $[2-4,10,13]$; if $f(z)=$ $a(1+\exp [-k-p(b-c) z])^{-1}$ where $a$ and $p$ are nonnegative numbers and $0<b<c$, the system (1) is a nerve equation studied in [1]. For each of these two systems, we give the following condition for the unique equilibrium to be globally asymptotically stable.

Corollary 3. If $f(z)=\left(1+z^{m}\right)^{-1}$ for $m \geq 1$ and

$$
S_{1} S_{2}-S_{3}+f^{\prime}(\sqrt[m]{(m-1) /(m+1)})>0,
$$

then the unique equilibrium of (1) is globally asymptotically stable in $\mathbf{R}_{+}^{3}$.

Corollary 4. If $f(z)=a(1+\exp [-k-p(b-c) z])^{-1}$ where $0<b<c$ and $S_{1} S_{2}-S_{3}+\frac{1}{4} a p(b-c)>0$, then the unique equilibrium of (1) is globally asymptotically stable in $\mathbf{R}_{+}^{3}$.

Remark 1. For positive feedback loop, Selgrade's theorems in [11, 12] and Hirsch's result in [6] are contained in Corollary 2.

Remark 2. Othmer [10] studied the asymptotic behavior of the flow generated by the vector field $F(x, y, z)=\left(-k_{1} x+\left(1+K_{1} z^{m}\right)^{-1}, k_{1} x-k_{2} y, k_{2} y-k_{3} z\right)$. He proved that for fixed $m$, when $k_{1}=k_{2}$ and either $K_{1}$ is sufficiently small or $k_{3}$ is sufficiently large, the unique equilibrium is globally asymptotically stable (see [10, Propositions 1-5]). We can easily transform this system into (1) with $f(z)=\left(1+z^{m}\right)^{-1}, a=k_{1} \mu, b=k_{2} \mu$, and $c=k_{3} \mu$ where $\mu=$ $\left(k_{1} k_{2} K_{1}^{1 / m}\right)^{-1 / 3}$. The above-mentioned result of Othmer follows immediately from Corollary 3.

Remark 3. Let $F(x, y, z)=\left(-a x+\left(1+z^{m}\right)^{-1}, x-b y, y-c z\right)$, and denote by $u$ the unique equilibrium of Hastings [4, p. 514], which is stated by the following proposition: Suppose $a b c<1$. Then there is a $m_{0}$ such that $D F(u)$ has two eigenvalues with positive real parts whenever $m>m_{0}$. However, this is not true if $a, b$, and $c$ are allowed to vary with $m$. For example, if we put $a=b=m$ and $c=\left(2 m^{2}\right)^{-1}$, then we can easily prove that $u$ is 
globally asymptotically stable for sufficiently large $m$ by Corollary 3 ; however, $a b c=\frac{1}{2}<1$.

Remark 4. Theorem 1 can be applied to the system that is neither positive feedback loop nor negative feedback loop. For example, let $a=b=c=1$ and $f(z)=\frac{1}{2}(3-\cos 2 z)$ in (1). We can prove that $\mathbf{R}_{+}^{3}$ is positively invariant and every trajectory in $\mathbf{R}_{+}^{3}$ is bounded. It is easy to show that $S_{1} S_{2}-S_{3}+f^{\prime}(z)>0$ and that (1) has a unique equilibrium in $\mathbf{R}_{+}^{3}$. Applying Theorem 1 we know that the unique equilibrium is globally asymptotically stable in $\mathbf{R}_{+}^{3}$; however, $f^{\prime}(z)=\sin 2 z$, which changes sign on $[0, \infty)$.

\section{THE PROOF OF RESULTS}

Proof of Theorem 1. Let $L=\{t(b c, c, 1): t \geq 0\}$ and $E$ be the set of equilibria for (1). It is easy to see that $E \subset L$. In order to construct a Liapunov function, we shall make a change of coordinates that takes $L$ to the $u$-axis; therefore,

$$
u=z \quad v=y-c z \quad w=x+a y-(a+b) c z .
$$

Transforming (1) by (2), we obtain

$$
\dot{u}=v \quad \dot{v}=w-S_{1} v \quad \dot{w}=-S_{3} u-S_{2} v+f(u) .
$$

We construct the Liapunov function,

$$
V(u, v, w)=\frac{1}{2}\left(S_{1} S_{3} u^{2}+2 S_{3} u v+S_{2} v^{2}+w^{2}\right)-v f(u)-S_{1} \int_{0}^{u} f(x) d x .
$$

Differentiating $V$ along a solution of (3) yields

$$
\frac{d V}{d t}=-\left(S_{1} S_{2}-S_{3}+f^{\prime}(u)\right) v^{2} \leq 0
$$

If $(u(t), v(t), w(t))$ is a bounded solution of (3), then the symbol of limit $v(t)=0$ by LaSalle's invariance principle (see [9, Theorem 2]). Denote by $\Omega$ the limit set of the solution; $\Omega$ is a compact, connected subset of the plane $\{v=0\}$. For any $\left(u_{0}, v_{0}, w_{0}\right) \in \Omega,(3)$ has one solution $\left(u_{0}(t), v_{0}(t), w_{0}(t)\right)$ with initial point $\left(u_{0}, v_{0}, w_{0}\right)$ defined on $\mathbf{R}$ such that $\left(u_{0}(t), v_{0}(t), w_{0}(t)\right) \in \Omega$ for $t \in \mathbf{R}$. Hence, $v_{0}(t) \equiv 0, u_{0}(t) \equiv u_{0}$, and $w_{0}(t) \equiv 0$, that is, $\Omega$ consists of equilibria of (3). Equivalently, every bounded solution of (1) approaches the set of equilibria.

We write $p \leq q(p<q)$, where $p$ and $q$ are vectors in $\mathbf{R}^{3}$, in case the specified inequality holds componentwise. If $p$ and $q$ are two vectors with $p<q$, let $[p, q]=\{r: p \leq r \leq q\}$.

Let $\Omega$ be an $\omega$-limit set of a bounded solution $(x(t), y(t), z(t))$ for (1), as just proved, $\Omega \subset L \cap E$. We claim that $\Omega$ only contains one point. If not, it follows from the compactness of $\Omega$ that there are maximal and minimal elements $p, q$ of $\Omega$ under the partial order $\leq$. Hence, $p<q$. Let $p=$ $\left(x_{1}, y_{1}, z_{1}\right)$ and $q=\left(x_{2}, y_{2}, z_{2}\right)$. Denote by $l$ the closed line segment with endpoints $p$ and $q$. By the connectedness of $\Omega$, we can easily prove that $\Omega=i$. Equivalently, $f(z)=S_{3} z$ for $z_{1} \leq z \leq z_{2}$. Since $f^{\prime}(z)=S_{3}>0$ for $z_{1} \leq z \leq z_{2}$, following Hirsch [5], (1) is a cooperative system on $[p, q]$. The well-known Kamke theorem (see [5, p. 425]) tells us that if $r_{1}, r_{2} \in[p, q]$ with $r_{1}<r_{2}$ and $r_{i}(t) \in[p, q]$ for all $t>0(i=1,2)$ then $r_{1}(t)<r_{2}(t)$ for 
all $t>0$, where $r_{i}(t)$ is the solution of (1) with initial point $r_{i} \quad(i=1,2)$. Therefore, it follows from $i \subset E$ that $[r, q]$ is a positively invariant set for any $r \in l$. Choose a point $r_{0} \in l$ such that $p<r_{0}<q$. Since $q \in \Omega$, there exists $t_{0}>0$ such that $\left(x\left(t_{0}\right), y\left(t_{0}\right), z\left(t_{0}\right)\right) \in\left[r_{0}, q\right]$. Because $\left[r_{0}, q\right]$ is positively invariant, $(x(t), y(t), z(t)) \in\left[r_{0}, q\right]$ for $t \geq t_{0}$, which implies that $\Omega \subset\left[r_{0}, q\right]$, contradicting $\Omega=i$. This contradiction shows our claim, that is, $(x(t), y(t), z(t))$ converges as $t \rightarrow \infty$. This completes the proof.

Remark. If $V(u, v, w) \rightarrow \infty$ as $u^{2}+v^{2}+w^{2} \rightarrow \infty$, every solution of (1) in $\mathbf{R}_{+}^{3}$ is bounded.

Proof of Corollary 2. It follows from (A2) that $\mathbf{R}_{+}^{3}$ is positively invariant. Since $S_{1} S_{2}-S_{3}>0$ and $f^{\prime}(z) \geq 0$ for $z \geq 0, S_{1} S_{2}-S_{3}+f^{\prime}(z)>0$. In order to prove Corollary 2 , by Theorem 1 , it suffices to prove that every solution of (1) in $\mathbf{R}_{+}^{3}$ is bounded if and only if $f\left(z_{k}\right) \leq S_{3} z_{k}$ for some sequences $z_{k} \rightarrow \infty$.

The vector field of $(1)$ is denoted by $F$. We write $\Phi=(b c, c, 1)$. If for sequences $z_{k} \rightarrow \infty, f\left(z_{k}\right) \leq S_{3} z_{k}$, then by calculation $F\left(z_{k} \Phi\right) \leq 0$. It follows from [11, Lemma 2.3] that the positive orbit of $z_{k} \Phi$ is bounded and its $\omega$-limit set is an equilibrium. By the well-known Kamke theorem, every solution of (1) is bounded. Conversely, there is a $Z$ such that $f(z)>S_{3} z$ for $z>Z$. This implies that $F(z \Phi) \supsetneqq 0$ for $z>Z$. It is easy to see that the set of equilibria for (1) is bounded. Without loss of generality, we may assume that all equilibria of (1) are contained in [0,Z]. Therefore, it follows from [11, Lemma 2.3] that the positive orbit of $z$ is unbounded for $z>Z$. The proof is complete.

Proof of Corollary 3. By Theorem 1, to complete the proof it suffices to show that $f^{\prime}(z)>f^{\prime}(\sqrt[m]{(m-1) /(m+1)})$ for $z \neq \sqrt[m]{(m-1) /(m+1)}$.

By calculation, we know that $f^{\prime}(z)=-m z^{m-1}\left(1+z^{m}\right)^{-2}$ and $f^{\prime \prime}(z)=$ $-m z^{m-2}\left(1+z^{m}\right)^{-3}\left[m-1-(m+1) z^{m}\right]$. Hence, when $z=\sqrt[m]{(m-1) /(m+1)}$, $f^{\prime}(z)$ has minimum value, i.e., $f^{\prime}(z)>f^{\prime}(\sqrt[m]{(m-1) /(m+1)})$ for $z \neq$ $\sqrt[m]{(m-1) /(m+1)}$. This completes the proof.

Proof of Corollary 4. By Theorem 1, to complete the proof it suffices to show that $f^{\prime}(z) \geq \frac{1}{4} a p(b-c)$.

By calculation we see that

$$
f^{\prime}(z)=a p(b-c) \exp [-k-p(b-c) z](1+\exp [-k-p(b-c) z])^{-2} .
$$

It is easy to see that

$$
x(1+x)^{-2} \leq \frac{1}{4} \text { for } x \geq 0 .
$$

So we immediately obtain that

$$
f^{\prime}(z) \geq \frac{1}{4} a p(b-c) \text { for } z \geq 0 .
$$

This proves Corollary 4.

\section{ACKNOWLEDGMENT}

The author is greatly indebted to the referee for valuable suggestions and comments. 


\section{REFERENCES}

1. U. an der Heiden, Existence of periodic solutions of a nerve equation, Biol. Cybern. 21 (1976), 37-39.

2. B. C. Goodwin, Oscillatory behavior in enzymatic control processes, in "Advances in Enzyme Regulation, vol. 3" (G. Weber, Ed.), Pergamon, Oxford, 1965.

3. J. S. Griffith, Mathematics of cellular control processes, I. Negative feedback to one gene, J. Theoret. Biol. 20 (1968), 202-208.

4. S. P. Hastings, On the uniqueness and global asymptotic stability of periodic solutions for a third order system, Rocky Mountain J. Math. 7 (1977), 513-538.

5. M. W. Hirsch, Systems of differential equations which are competitive or cooperative II: Convergence almost everywhere, SIAM J. Math. Anal. 16 (1985), 423-439.

6. __ Systems of differential equations which are competitive or cooperative V: Convergence in 3-dimensional systems, J. Differential Equations 80 (1989), 94-106.

7. Jiang $\mathrm{Ji}-\mathrm{Fa}$, On the asymptotic behavior of a class of nonlinear differential equations, Nonlinear Anal. Theory, Methods and Applications 14 (1990), 453-467.

8. __ The asymptotic behavior of a class of second-order differential equations with applications to electrical circuit equations, J. Math. Anal. Appl. 149 (1990), 26-37.

9. J. P. LaSalle, Stability theory for ordinary differential equations, J. Differential Equations 4 (1968), 57-65.

10. H. G. Othmer, The qualitative dynamics of a class of biochemical control circuits, J. Math. Biol. 3 (1976), 53-78.

11. J. F. Selgrade, Asymptotic behavior of solutions to single loop positive feedback systems, J. Differential Equations 38 (1980), 80-103.

12. _ Mathematical analysis of a cellular control process with positive feedback, SIAM J. Appl. Math. 36 (1979), 219-229.

13. J. J. Tyson, On the existence of oscillatory solutions in negative feedback cellular control processes, J. Math. Biol. 1 (1975), 311-315.

Department of Mathematics, Anhui Normal University, Wuhu, Anhui Province, PeoPLE'S REPUBLIC OF CHINA 